Methods: Eligible patients, aged $\geq 18$ years, with active moderate-to-severe RA (ACR/EULAR 2010 criteria) who were IV abatacept-naive and initiated SC abatacept $125 \mathrm{mg}$ once weekly, were enrolled into two cohorts: biologic (b) DMARD-naive patients and those with $\geq 1$ prior bDMARD treatment failure. This post hoc analysis assessed the mean change in disease activity (CDAI, SDAI and DAS28 [ESR]) from baseline (BL) at 6, 12, 18 and 24 months in response to treatment with abatacept. Patients were stratified by BL serostatus (all patients, RF/ACPA double positive [+/+] RA; RF/ACPA single positive [+/-; RF+/ACPA- or $\mathrm{RF}-/ \mathrm{ACPA}+] \mathrm{RA}$ and RF/ACPA double negative [-/-] RA) and by line of therapy (all patients, patients receiving abatacept as a first-line or $\geq$ second-line therapy and those receiving abatacept following 1 or $\geq 2$ prior bDMARDs). Overall patient data, as well as data for patients who were + /- or those who had 1 or $\geq 2$ previous bDMARDs, are not shown. Estimates of mean difference are from $t$-test.

Results: Among 2892 eligible patients in ASCORE, 1748 patients with RF/ACPA status available at BL were included in this analysis (1079 +/+ RA, $326+/-$ RA and $343-/-$ RA). After 6 months, patients with +/+ RA on first-line abatacept therapy had better improvements in CDAI and SDAI scores from BL than patients on $\geq$ second-line abatacept therapy (mean difference $[95 \% \mathrm{CI}]:-3.4[-5.6,-1.1]$; $\mathrm{p}=0.0032$ and $-3.9[-6.5,-1.3] ; \mathrm{p}=0.0035$, respectively); better improvements in SDAl were also seen after 12 months (mean difference $[95 \% \mathrm{Cl}]$ : $-3.5[-6.5$ -0.5 ]; $p=0.0207$ ). Changes in CDAI and SDAI scores were comparable after 18 and 24 months. At 6 and 12 months, patients with +/+ RA on first-line therapy had better improvements from BL in DAS28 (ESR) than those on $\geq$ second-line therapy (mean differences $[95 \% \mathrm{Cl}]:-0.5[-0.8,-0.2] ; \mathrm{p}=0.0002$ and $-0.4[-0.7$, $-0.0] ; p=0.0317$, respectively); changes were comparable at 18 and 24 months (Figure 1). For patients on $\geq$ second-line therapy, at 18 months those with $+/+$ RA had better improvements from BL in DAS28 (ESR) than those with -/- RA (mean difference $[95 \% \mathrm{Cl}]:-0.7[-1.2,-0.1] ; p=0.0232$ ). For patients not stratified by line of therapy, changes in DAS28 (ESR) were comparable between the $+/+$ and -/- RA subgroups over time, with the exception of 6 months where patients with -/- RA had better improvements from BL compared with patients with +/+ RA (mean difference [95\% Cl]: $-0.3[-0.6,-0.0] ; p=0.0495$ ).

Conclusion: In this real-world, post hoc analysis, patients with +/+ RA who received abatacept as a first-line therapy had greater early improvements in disease activity compared with patients who received abatacept as a $\geq$ second-line therapy. Improvements in disease activity at 24 months were comparable between patients who were $+/+$ and those who were $-/-$. Larger studies are needed to further corroborate these findings.

REFERENCES:

[1] Katchamart W, et al. Rheumatol Int 2015;35:1693-1699.

[2] Malmström V, et al. Nat Rev Immunol 2017;17:60-75

[3] Alten R, et al. Ann Rheum Dis 2019;78(Suppl 2):A1639.

Figure 1. Mean change from BL in DAS28 (ESR) for patients with RA treated with abatacept

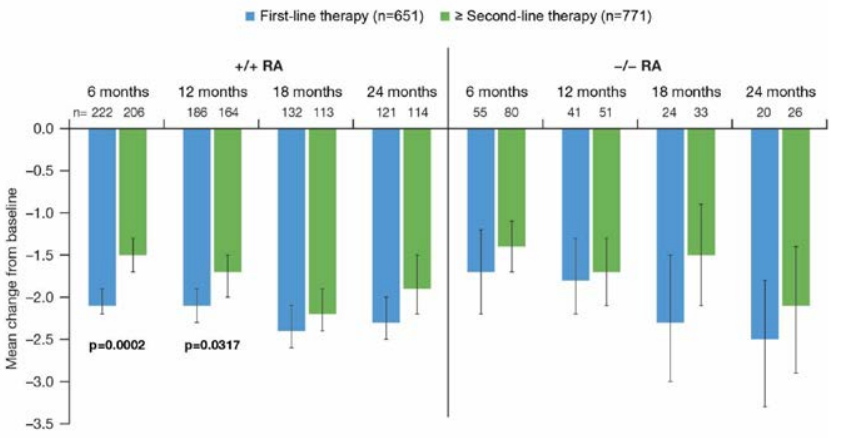

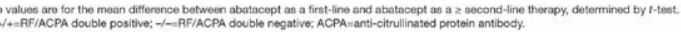

Acknowledgements: Professional medical writing and editorial assistance was provided by Rachel Rankin, PhD, at Caudex and was funded by Bristol Myers Squibb. This study was funded by Bristol Myers Squibb.

Disclosure of Interests: Rieke Alten Speakers bureau: AbbVie, Bristol Myers Squibb, Gilead, Janssen, Lilly, Pfizer, Consultant of: AbbVie, Bristol Myers Squibb, Gilead, Janssen, Lilly, Pfizer, Grant/research support from: AbbVie, Bristol Myers Squibb, Gilead, Janssen, Lilly, Pfizer, Xavier Mariette Consultant of: Bristol Myers Squibb, Galapagos, Gilead, GlaxoSmithKline, Janssen, Pfizer, UCB, Rene-Marc Flipo Speakers bureau: AbbVie, Bristol Myers Squibb, Janssen, Lilly, Medac, Merck Sharp \& Dohme, Novartis, Pfizer, Roche-Chugai, Grant/research support from: Amgen, Janssen, Novartis, Pfizer, Roberto Caporali Speakers bureau: AbbVie, Amgen, Bristol Myers Squibb, Celltrion, Fresenius Kabi, Galapagos, Gilead, Lilly, Merck Sharp \& Dohme, Pfizer, Roche, Samsung Bioepis, Sanofi, UCB, Consultant of: Galapagos, Gilead, Janssen, Lilly, Merck Sharp \& Dohme, Maya H Buch Speakers bureau: AbbVie, Consultant of: AbbVie, Eli Lilly, Gilead, Merck Serono, Pfizer, Roche, Sanofi,
Grant/research support from: Gilead, Pfizer, Roche, UCB, Yusuf Patel: None declared, Raimón Sanmartí Speakers bureau: AbbVie, Bristol Myers Squibb, Gebro, Janssen, Lilly, Merck Sharp \& Dohme, Pfizer, Roche, Sanofi, Consultant of: AbbVie, Bristol Myers Squibb, Gebro, Lilly, Merck Sharp \& Dohme, Pfizer, Roche, Sanofi, Grant/research support from: Bristol Myers Squibb, Merck Sharp \& Dohme, Pfizer, Sara Marsal Speakers bureau: Bristol Myers Squibb, Celgene, Pfizer, Roche, Sanofi, UCB, Consultant of: AbbVie, Bristo Myers Squibb, Celgene, Galapagos, Merck Sharp \& Dohme, Pfizer, Roche Sanofi, UCB, Grant/research support from: AbbVie, Bristol Myers Squibb Celgene, Janssen, Merck Sharp \& Dohme, Novartis, Pfizer, Roche, Sanofi, UCB, M.T. Nurmohamed Speakers bureau: AbbVie, Bristol Myers Squibb, Eli Lilly, Roche, Sanofi, Consultant of: AbbVie, Celgene, Celltrion, Eli Lilly, Jans sen, Grant/research support from: AbbVie, Bristol Myers Squibb, Celgene, Eli Lilly, Janssen, Merck Sharp \& Dohme, Mundipharma, Novartis, Pfizer, Roche Sanofi, Hedley Griffiths Consultant of: AbbVie, Gilead, Janssen, Novartis, Peter Peichl: None declared, Bettina Bannert: None declared, Adrian Forster: None declared, Melanie Chartier Shareholder of: Bristol Myers Squibb, Employee of: Bristol Myers Squibb, Yedid Elbez Consultant of: Bristol Myers Squibb, Christiane Rauch Shareholder of: Bristol Myers Squibb, Employee of: Bristol Myers Squibb, Karissa Lozenski Shareholder of: Bristol Myers Squibb, Employee of: Bristol Myers Squibb, Vadim Khaychuk Shareholder of: Bristol Myers Squibb, Employee of: Bristol Myers Squibb DOI: 10.1136/annrheumdis-2021-eular.928

\section{POS0600 \\ IMMUNOGENICITY OF RITUXIMAB BIOSIMILAR GP2013: A RARE EVENT, BUT NOT WITHOUT CONSEQUENCES}

J. Avouac ${ }^{1}$, R. Cougnaud Murail ${ }^{1}$, C. Goulvestre ${ }^{2}$, S. Dumas ${ }^{3}$, A. Moltó $^{1}$, C. Miceli Richard ${ }^{1}$, O. Conort ${ }^{3}$, F. Batteux ${ }^{2}$, Y. Allanore ${ }^{1} .{ }^{1}$ Cochin Hospital, Rheumatology Department, Paris, France; ${ }^{2}$ Cochin Hospital, Immunology Department, Paris, France; ${ }^{3}$ Cochin Hospital, Pharmacy Department, Paris, France

Background: The bioequivalence between rituximab (RTX) originator and its biosimilar GP2013 has been demonstrated in rheumatoid arthritis (RA) (1). A recent randomized controlled trial suggested in a selected population a very low immunogenicity of GP2013 in RA (<1\%) (2).

Objectives: To study in daily practice the risk of immunogenicity of patients treated with GP2013 for their chronic inflammatory rheumatic disorder.

Methods: Prospective routine care study carried out between September 2018 and August 2020 in the Rheumatology department of Cochin Hospital. We consecutively included patients treated with the biosimilar RTX GP2013, systematically used in the department since March 2018. Samples were taken before each infusion in order to detect anti-RTX antibodies $(\mathrm{Ab})$ and RTX residual concentrations by ELISA (Lisa Tracker Duo Rituximab, LTR005, Theradiag).

Results: We included 159 consecutive patients treated with GP2013 (124 women $78 \%$ ) with a mean age of $59 \pm 13$ years and a mean disease duration of $18 \pm 11$ years. Among these 159 patients, 108 (68\%) had RA and 51 had another disease (16 systemic sclerosis (SSc), 15 mixed connective tissue disease (MCTD), 5 systemic lupus (SLE), 5 inflammatory myopathies (MI), 5 undifferentiated polyarthritis, 2 juvenile idiopathic arthritis (JIA) and 3 primary Sjögren's syndromes). 137 patients (86\%) were receiving associated disease-modifying therapy (DMARD), mainly methotrexate $(111 / 137$ patients, $81 \%) .120$ patients $(75 \%)$ were in maintenance therapy with originator RTX (cumulative dose of RTX: $3.5 \pm 6 \mathrm{~g}$ ) before the switch to GP2013 in March 2018. Originator RTX was not re-established during the entire treatment period. The other 39 patients (25\%) treated with GP2013 were naïve of originator RTX

The analysis of the first sample, performed before the second GP2013 infusion, identified 8 patients ( 5 RA, 1 SLE, 1 MCTD and 1 SSc) with positive anti-RTX antibodies (prevalence 5\%), with rates varying between 6 and $>100 \mathrm{ng} / \mathrm{mL}$ and undetectable residual RTX concentrations. Among these 8 patients, 6 had previously received originator RTX and 2 were RTX-naïve patients. There was a trend for higher body mass index in patients with positive anti-RTX antibodies $\left(28 \pm 7\right.$ vs. $\left.25 \pm 6 \mathrm{~kg} / \mathrm{m}^{2}, \mathrm{p}=0.12\right)$, and no association was observed between antiRTX immunization and age, disease duration, combination with conventional DMARD, mean interval between infusions or cumulative RTX dose.

Among the 8 immunized patients, two groups could be isolated: i) a group of 5 patients ( 3 RA, 1 SLE, 1 SSc) with low antibody levels $(6-22 \mathrm{ng} / \mathrm{mL})$ and no significant clinical consequences (absence of treatment discontinuation and loss of efficacy after $13 \pm 4$ months of follow-up, only one minor allergic reaction) and ii) a group of 3 patients ( 2 RA, 1 MCTD) with a high antibody levels ( $\geq 100 \mathrm{ng} /$ $\mathrm{mL}$ ) and meaningful clinical consequences: one severe allergic reaction during the second GP2013 infusion leading to treatment discontinuation, and a loss of efficacy with incomplete B depletion in 2 patients leading to RTX dose escalation from $500 \mathrm{mg}$ to $1 \mathrm{~g}$. Among the 151 patients not immunized at the time of the first sample, no severe allergic reaction and 6 minor allergic reactions were noted under GP2013. 
Conclusion: The immunogenicity of patients treated with RTX is a rare event with possible clinical and biological consequences, especially in patients with high antibody levels.

REFERENCES

[1] Smolen et al, Ann Rheum Dis 2017

[2] Tony et al, Arthritis Care Res 2019

Disclosure of Interests: None declared

DOI: 10.1136/annrheumdis-2021-eular.954

\section{POS0601 DIFFERENCES IN DRUG SURVIVAL BETWEEN ORIGINATOR AND BIOSIMILAR PRODUCTS AMONG FIRST USERS OF EACH MOLECULE}

D. Di Giuseppe ${ }^{1}$, H. Bower ${ }^{1}$, B. Delcoigne ${ }^{1}$, T. Frisell ${ }^{1}$, K. Chatzidionysiou ${ }^{1,2}$, U. Lindström ${ }^{3}$, C. Sjowall ${ }^{4}$, E. Lindqvist ${ }^{5}$, J. Askling ${ }^{1}$ on behalf of ARTIS. ${ }^{1}$ Karolinska Institute, Department of Medicine Solna, Stockholm, Sweden; ${ }^{2} \mathrm{Nya}$ Karolinska, Rheumatology Unit, Stockholm, Sweden; ${ }^{3}$ Sahlgrenska Academy, University of Gothenburg, Rheumatology Unit, Karolinska University Hospital, Gothenburg, Sweden; ${ }^{4}$ Linköping University, Division of Inflammation and Infection, Department of Biomedical and Clinical Sciences, Linköping, Sweden; ${ }^{5}$ Lund University, Skåne University Hospital, Department of clinical sciences, Rheumatology, Lund, Sweden

Background: Biosimilar products of biological disease-modifying antirheumatic drugs (bDMARDs) entered the Swedish market in 2015, with regulatory approvals based on head to head trials of limited duration. Longer-term comparative drug survival, in clinical practice, remains less well documented.

Objectives: To compare survival on drug between biosimilars and their originator products among first starters of etanercept, infliximab, adalimumab and rituximab.

Methods: Data from the Swedish Rheumatology Quality register (SRQ) was used to identify and follow patients who started a first ever treatment with etanercept since April 2015 (originator=ETA, biosimilar= SB4), infliximab since March 2014 (originator=IFX,biosimilar= CT-P13), adalimumab since January 2018 (originator=ADA biosimilars=SB5, ABP501), or rituximab since January 2018 (originator=RIT, biosimilar= GP2013), through December $31^{\text {st }}, 2019$, date of first discontinuation of the drug, or death. Discontinuation was defined as lack of effectiveness or adverse events, while other reasons for interruption of the drug (including non-medical switch) were considered censoring events. Descriptive characteristics were collected from the SRQ and tabulated. Hazard ratios (HR) of discontinuation were estimated using Cox regression, with each drug analyzed separately, adjusted for age,sex, indication, line of treatment,disease duration,year of treatment start,region and concomitant use of csDMARD.

Results: 9274 patients started etanercept(49\% SB4), 3609 started infliximab(64\% CT-P13), 3117 started adalimumab(27\% SB5, 14\% ABP 501), and 763 started rituximab(39\% GP2013), Table 1. Patients starting CT-P13 and GP2013 were less likely to be biologics-naive compared to those starting the originator product. Initiators of SB5,ABP501 and GP2013 were more likely, and those starting CT-P13 were less likely,to be on concomitant csDMARDs compared to those starting the originator products. Patients characteristics of ETA and SB4 were similar.

The introduction of a biosimilar was typically followed by a decrease in the uptake of the originator, but for ETA a change in pricing in 2018 later led to a reversal of this pattern (Figure 1).

For IFX,ADA, and RIT, survival on drug was similar for the originator and its biosimilar(s). For ETA,risk of discontinuation was somewhat lower for the biosimilar than for the originator(adjusted HR:0.87,95\% confidence interval:0.79-0.95), Table 1.

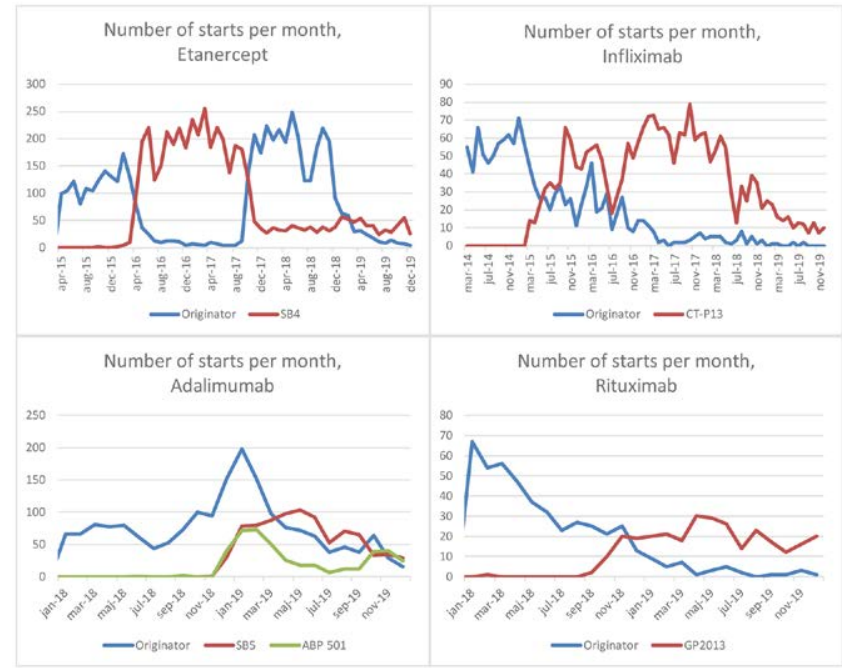

Figure 1. Number of starts of biosimilars compared to the originator during the follow-up time, by molecule

Conclusion: Despite their identical indications and therapeutic positioning, there are some differences in the baseline characteristics between patients who start ADA, IFX and RIT and their biosimilars. There are no differences in drug survival between originator and biosimilar with the possible exception of etanercept although the observed difference should be interpreted in light of possible unmeasured or residual channeling

Disclosure of Interests: Daniela Di Giuseppe: None declared, Hannah Bower: None declared, Bénédicte Delcoigne: None declared, Thomas Frisell: None declared, Katerina Chatzidionysiou Consultant of: Eli Lilly, AbbVie and Pfizer, Ulf Lindström: None declared, Christopher Sjowall: None declared, Elisabet Lindqvist: None declared, Johan Askling Grant/research support from: Abbvie, AstraZeneca, BMS, Eli Lilly, MSD, Pfizer, Roche, Samsung Bioepis, Sanofi, and UCB DOI: 10.1136/annrheumdis-2021-eular.977

\section{POS0602 ROLE OF INTRAARTICULAR BIOLOGICS AND METHOTREXATE IN THE MANAGEMENT OF ACTIVE INFLAMMATORY MONOARTHRITIS}

F. Aslam ${ }^{1}$, M. Meyer ${ }^{1}$, M. Pham ${ }^{2} .{ }^{1}$ Mayo Clinic, Rheumatology, Scottsdale, United States of America; ${ }^{2}$ Mayo Clinic, Medicine, Scottsdale, United States of America

Background: Expansion of immunosuppressive medications has greatly improved disease activity control in rheumatoid arthritis (RA) patients. However, persistent symptomatic monoarthritis can be a problem in some patients who otherwise have satisfactory disease control. Intra-articular (IA) corticosteroid (CS) injections have remained a treatment cornerstone for these patients, but the need for other therapies is clear. Patients can fail such therapy, may develop adverse effects or have comorbidities that are exacerbated by IA CS. $50 \%$ of patients relapse after IA CS therapy. Several studies have looked at other agents such as methotrexate (MTX) or tumor necrosis factor inhibitors (TNF-i) as IA treatment options.

Objectives: To systematically review the evidence regarding utility of IA methotrexate and biologics in managing inflammatory monoarthritis in patients with RA

Table 1. Hazard ratios of discontinuation and descriptive characteristics of biosimilar vs. originator among first starters of each molecule, until $31^{\text {st }}$ December 2019

\begin{tabular}{|c|c|c|c|c|c|c|c|c|c|}
\hline & \multicolumn{2}{|l|}{ Etanercept } & \multicolumn{2}{|l|}{ Infliximab } & \multicolumn{3}{|c|}{ Adalimumab } & \multicolumn{2}{|l|}{ Rituximab } \\
\hline & Originator & SB4 & Originator & CT-P13 & Originator & SB5 & ABP 501 & Originator & GP2013 \\
\hline $\mathrm{N}$ & 4721 & 4553 & 1308 & 2301 & 1834 & 852 & 431 & 465 & 298 \\
\hline Discontinuation & 1289 & 1236 & 582 & 878 & 399 & 139 & 80 & 57 & 26 \\
\hline Adjusted hazard ratios* & Ref & $0.87(0.79-0.95)$ & Ref & $1.14(0.99-1.31)$ & Ref & $1.02(0.83-1.26)$ & $1.16(0.88-1.52)$ & Ref & $1.12(0.68-1.85)$ \\
\hline Age, mean years (std) & $51(16)$ & $51(15)$ & $49(16)$ & $49(16)$ & $48(15)$ & $52(15)$ & $51(15)$ & $59(15)$ & $60(15)$ \\
\hline Female, $\%$ & $67 \%$ & $65 \%$ & $61 \%$ & $64 \%$ & $62 \%$ & $64 \%$ & $65 \%$ & $75 \%$ & $76 \%$ \\
\hline RA, \% & $46 \%$ & $48 \%$ & $39 \%$ & $35 \%$ & $33 \%$ & $42 \%$ & $43 \%$ & $61 \%$ & $76 \%$ \\
\hline Bionaïve, \% & $72 \%$ & $72 \%$ & $76 \%$ & $69 \%$ & $45 \%$ & $52 \%$ & $43 \%$ & $53 \%$ & $38 \%$ \\
\hline Disease duration, mean years (std) & $11(12)$ & $11(11)$ & $11(11)$ & $11(11)$ & $12(13)$ & $12(11)$ & $14(15)$ & $14(19)$ & $15(11)$ \\
\hline DAS28, mean & $4.0(1.3)$ & $4.0(1.4)$ & $4.1(1.4)$ & $4.1(1.4)$ & $3.7(1.4)$ & $3.8(1.3)$ & $4.0(1.3)$ & $4.5(1.4)$ & $4.7(1.4)$ \\
\hline Concomitant csDMARDs, $\%$ & $45 \%$ & $47 \%$ & $57 \%$ & $48 \%$ & $37 \%$ & $49 \%$ & $42 \%$ & $36 \%$ & $43 \%$ \\
\hline
\end{tabular}

Abbreviations: RA=rheumatoid arthritis. csDMARDs=conventional synthetic DMARD, std=standard deviation. 